\title{
Outcomes of patients with stage III non-small cell lung cancer (NSCLC) that harbor a STK11 mutation
}

\author{
Josiah An ${ }^{1} \wedge$, Melissa $\operatorname{Yan}^{2} \wedge$, Nanmeng Yu ${ }^{1}$, Adithya Chennamadhavuni ${ }^{1}$, Muhammad Furqan ${ }^{1}$, \\ Sarah L. Mott ${ }^{3} \wedge$, Bradley T. Loeffler ${ }^{3} \wedge$, Timothy Kruser ${ }^{4}$, Timothy L. Sita ${ }^{5}$, Lawrence Feldman ${ }^{6}$, \\ Ryan Nguyen ${ }^{6}$, Mary Pasquinelli $^{6}$, Nasser H. Hanna ${ }^{2}$, Taher Abu Hejleh ${ }^{1}$
}

${ }^{1}$ Division of Hematology, Oncology, Blood \& Marrow Transplantation, University of Iowa Hospitals and Clinics, Iowa City, IA, USA; ${ }^{2}$ Division of Hematology and Oncology, Indiana University Health - Melvin and Bren Simon Cancer Center, Indianapolis, IN, USA; ${ }^{3}$ Holden Comprehensive Cancer Center, University of Iowa, Iowa City, IA, USA; ${ }^{4}$ Turville Bay Radiation Oncology, SSM Health, Madison, WI, USA; ${ }^{5}$ Department of Radiation Oncology, Northwestern Memorial Hospital, Chicago, IL, USA; ${ }^{6}$ Division of Hematology and Oncology, The University of Illinois at Chicago, Chicago, IL, USA

Contributions: (I) Conception and design: J An, M Furqan, NH Hanna, T Abu Hejleh; (II) Administrative support: J An; (III) Provision of study materials or patients: J An, M Furqan, T Abu Hejleh; (IV) Collection and assembly of data: J An, M Yan, N Yu, T Kruser, TL Sita, L Feldman, R Nguyen, M Pasquinelli, NH Hanna, T Abu Hejleh; (V) Data analysis and interpretation: J An, SL Mott, BT Loeffler, T Abu Hejleh; (VI) Manuscript writing: All authors; (VII) Final approval of manuscript: All authors.

Correspondence to: Taher Abu Hejleh, MD. Division of Hematology, Oncology, Blood \& Marrow Transplantation, Department of Internal Medicine, University of Iowa Hospitals and Clinics, 200 Hawkins Drive, C32 GH, Iowa City, IA 52242, USA. Email: Taher-Hejleh@uiowa.edu.

Background: STK11 mutation $\left(S T K 11^{m}\right)$ in patients (pts) with stage IV non-small cell lung cancer (NSCLC) is associated with inferior survival and poor response to immune checkpoint inhibitors (ICI). The significance of $S T K 11^{m}$ in stage III NSCLC pts treated with concurrent chemoradiation (CCRT) with or without consolidation ICI is unknown.

Methods: Stage III NSCLC patients who received CCRT and had known STK11 mutational status were included in this retrospective study. The data on the $S T K 11^{m}$ pts were collected from 4 cancer institutions. A cohort of pts with wild type $S T K 11\left(S T K 11^{w}\right)$ from the University of Iowa served as a comparison group. Patient demographics and clinical characteristics were collected. Cox regression models were used to explore the effect of STK11 mutation on survival.

Results: 75 pts with stage III NSCLC who had known STK11 mutational status were identified. 16/75 (21\%) had $S T K 11^{m}$. 5/16 with $S T K 11^{m}$ did not receive CCRT so they were excluded from the analysis. The clinical and demographic characteristics for the $11 S T K 11^{m}$ and $59 S T K 11^{w}$ pts were not statistically different $\left(S T K 11^{m}\right.$ vs. STK11 $\left.1^{w}\right)$ : mean age: 57 vs. 64 yrs, non-squamous histology: 8/11 (73\%) vs. 37/59 (63\%), KRAS mutation: $3 / 11$ (27\%) vs. $11 / 59$ (19\%), TP53 mutation: 6/11 (55\%) vs. 15/59 (25\%), PD-L1 $\geq 50 \%$ : 1/8 (13\%) vs. 10/32 (31\%), and consolidation ICI 6/11 (55\%) vs. $17 / 59$ (29\%). Regarding the 6 STK1 $1^{m}$ pts who received ICI (4 pembrolizumab, 2 durvalumab), the median number of ICI infusions was 8 (range, 3-17) vs. 6 (range, 1-25) in the 17 pts with $S T K 11^{w}$ who received ICI (durvalumab). After adjusting for performance status and cancer stage, multivariable analysis showed that progression free survival (PFS) for the $S T K 11^{m}$ pts was significantly worse than $S T K 11^{\mathrm{w}}$ pts $(\mathrm{HR}=2.25$; 95\% CI, 1.03-4.88, $\mathrm{P}=0.04)$, whereas overall survival (OS) showed no significant difference for $S T K 11^{m}$ vs. $S T K 11^{w}$ patients (HR 1.47, 95\% CI, 0.49-4.38, P=0.49).

Conclusions: In stage III NSCLC patients who received CCRT, STK1 $1^{m}$ was associated with worse PFS compared to $S T K 11^{w}$. Larger studies are needed to further explore the prognostic implications of $S T K 11^{m}$ in stage III NSCLC and whether ICI impacts survival for this subgroup.

Keywords: Non-small cell lung cancer (NSCLC); immunotherapy; STK11; KRAS; TP53

^ ORCID: Josiah An, 0000-0002-6978-7388; Melissa Yan, 0000-0002-9102-0431; Sarah L. Mott, 0000-0001-9381-2080; Bradley L. Loeffler, 0000-0002-6006-2372; Lawrence Feldman, 0000-0002-8863-6649. 
Submitted Apr 04, 2021. Accepted for publication Aug 09, 2021.

doi: $10.21037 /$ tlcr-21-177

View this article at: https://dx.doi.org/10.21037/tlcr-21-177

\section{Introduction}

Unresectable stage III non-small cell lung cancer (NSCLC) in patients with good performance status is treated with concurrent platinum based chemotherapy with definitive dose radiation (CCRT) followed by the PD-L1 inhibitor, durvalumab (1). The addition of durvalumab to CCRT improved survival from $55.6 \%$ to $66.3 \%$ at 24 months (1). Although this improvement in survival is practice changing, a large number of stage III NSCLC patients still have a poor prognosis. In 2020, it is estimated there will be 229,000 new cases of lung cancer (2) of which stage III cancer is expected in approximately $30 \%$ of all NSCLC cases.

Serine/threonine kinase 11 (STK11), also known as liver kinase B1 (LKB1), is a gene found on chromosome 19p13. Germline mutation of STK11 is associated with PeutzJeghers Syndrome (PJS) (3). Patients with PJS can develop intestinal hamartomatous polyps and are more likely to develop malignancies such as gastrointestinal, testis, ovary, and breast cancers (3). In lung cancer, somatic mutations of STK11 are seen in up to $42 \%$ of NSCLC; however, most studies have shown a mutation frequency of approximately $20 \%$ (4). STK11 functions as a tumor suppressor gene and is involved in the activation of AMP-activated protein kinase (AMPK), which modulates cell glucose and lipid metabolism (5). More importantly, in-vivo studies indicate that STK11 is involved in the differentiation and metastases of lung cancer (6). STK11 inactivation leads to increase in pro-inflammatory cytokines such as CXCL7 and causes a shift in the microenvironment with neutrophil accumulation and decrease in $T$ cell lymphocytes $(7,8)$.

The effect of $S T K 11^{m}$ on outcomes of stage III NSCLC treated with curative intent is unknown. It is unclear whether STK $11^{m}$ affects prognosis of stage III NSCLC or predicts response to ICI consolidation after CCRT. In advanced stage NSCLC, STK1 $1^{m}$ has been associated with poor response to chemotherapy and ICI, and inferior survival outcomes (9-15). In this study, we sought to explore $S T K 11^{m}$ as a prognostic genetic alteration in stage III NSCLC patients managed with definitive chemoradiation +/- consolidative ICI. We present the following article in accordance with the REMARK reporting checklist (available at https://dx.doi.org/10.21037/tlcr-21-177).

\section{Methods}

The study was conducted in accordance with the Declaration of Helsinki (as revised in 2013). The informed consent from the patients were waived. The study was approved by institutional Review Boards (IRBs) of University of Iowa Hospitals and Clinics, Indiana University Health, Northwestern Memorial Hospital, and the University of Illinois at Chicago. The 4 institutions provided a total of 16 patients with stage III NSCLC known to have STK1 $1^{m}$ diagnosed between 2013 and 2019. Of these, 11 received CCRT and were included in the study. A comparison group of 59 patients with STK1 $1^{w}$ stage III NSCLC who received CCRT diagnosed between 2013 and 2019 were identified at University of Iowa and served as a comparison group. Stage III NSCLC, receiving any CCRT, and having a known STK11 mutational status were requirements for inclusion in the analysis in this study.

The patients' charts were reviewed for clinical and demographic characteristics. These included age, sex, smoking history, stage, ECOG performance status, histology, STK11, KRAS and TP53 status, PD-L1 expression, and treatment details including radiation, chemotherapy and immunotherapy. STK $11^{m}$ was identified by next generation sequencing of the tumor tissue using the platform of choice available in the institutions participating in this study. Specific STK11 locus alteration information was not collected. Information about other NSLCC driver mutations such as EGFR and $A L K$ were not collected.

\section{Statistical analysis}

Chi-squared or Fisher's exact tests were used to compare categorical variables, and Wilcoxon rank sum tests were used to compare continuous variables between STK11 wild type and mutant. Survival probabilities were estimated and plotted using the Kaplan-Meier method. Cox regression models were used to assess the effects of clinical, pathologic, and treatment variables on progression-free survival (PFS) and overall survival (OS). The time for PFS was calculated from the date of diagnosis until progression or death due to any cause. The time for OS was calculated from the date of diagnosis until death due to any cause. Estimated effects 
Table 1 Demographics and clinical data for $S T K 11^{m}$ and $S T K 11^{w}$ stage III NSCLC

\begin{tabular}{|c|c|c|c|c|c|}
\hline Variable & Level & Total, N=70, n (\%) & \multicolumn{3}{|c|}{ STK11, n (\%) } \\
\hline \multirow[t]{2}{*}{ Sex } & $F$ & $35(50.0)$ & $31(52.5)$ & $4(36.4)$ & 0.32 \\
\hline & $M$ & 35 (50.0) & $28(47.5)$ & 7 (63.6) & \\
\hline Age & $\leq 65$ years & 41 (58.6) & $32(54.2)$ & $9(81.8)$ & 0.11 \\
\hline \multirow[t]{2}{*}{ Smoking history } & Current & 65 (92.9) & $54(91.5)$ & $11(100)$ & 1.00 \\
\hline & Never smoker & $5(7.1)$ & $5(8.5)$ & $0(0)$ & \\
\hline \multirow[t]{2}{*}{ ECOG } & $0-1$ & $60(85.7)$ & $49(83.1)$ & $11(100)$ & 0.34 \\
\hline & $2-3$ & $10(14.3)$ & 10 (16.9) & $0(0)$ & \\
\hline \multirow[t]{2}{*}{ Stage } & IIIA & 38 (54.3) & $32(54.2)$ & $6(54.5)$ & 0.98 \\
\hline & $\mathrm{IIIB} / \mathrm{C}$ & $32(45.7)$ & $27(45.8)$ & $5(45.5)$ & \\
\hline \multirow[t]{2}{*}{ KRAS } & No & $56(80.0)$ & $48(81.4)$ & $8(72.7)$ & 0.68 \\
\hline & Yes & $14(20.0)$ & $11(18.6)$ & $3(27.3)$ & \\
\hline \multirow[t]{2}{*}{ TP53 } & No & $49(70.0)$ & $44(74.6)$ & $5(45.5)$ & 0.07 \\
\hline & Yes & $21(30.0)$ & $15(25.4)$ & $6(54.5)$ & \\
\hline \multirow[t]{2}{*}{ PD-L1 } & $<1 \%$ & $21(52.5)$ & $16(50.0)$ & $5(62.5)$ & 0.66 \\
\hline & $1-50 \%$ & $8(20.0)$ & $6(18.8)$ & $2(25.0)$ & \\
\hline \multirow{2}{*}{ Cycles of ICI } & Median & 0 & 0 & 3 & \\
\hline & Range & $(0-25)$ & $(0-25)$ & $(0-17)$ & \\
\hline
\end{tabular}

NSCLC, non-small cell lung cancer.

of predictors are reported as hazard ratios (HR) along with $95 \%$ confidence intervals. All statistical testing was twosided and assessed for significance at the $5 \%$ level using SAS v9.4 (SAS Institute, Cary, NC).

\section{Results}

\section{Patient demographics and clinical characteristics}

After excluding the 5 patients with $S T K 11^{\mathrm{m}}$ who did not receive CCRT, a total of 70 pts with stage IIIA-C NSCLC were included in the analysis; 11 patients with STK1 $1^{m}$ and 59 patients with STK11 ${ }^{w}$ (Table 1). When $S T K 11^{m}$ patients were compared to $S T K 11^{w}$, there was no significant difference in the gender distribution among the two groups. Numerically, the proportion of age $\leq 65$ years in the $S T K 11^{m}$ group compared to $S T K 11^{w}$ was higher but this was not statistically significant $(82 \%$ vs. $54 \%, \mathrm{P}=0.11)$. When all patients including the 5 with $S T K 11^{m}$ who did not receive CCRT were accounted for in the analysis, 


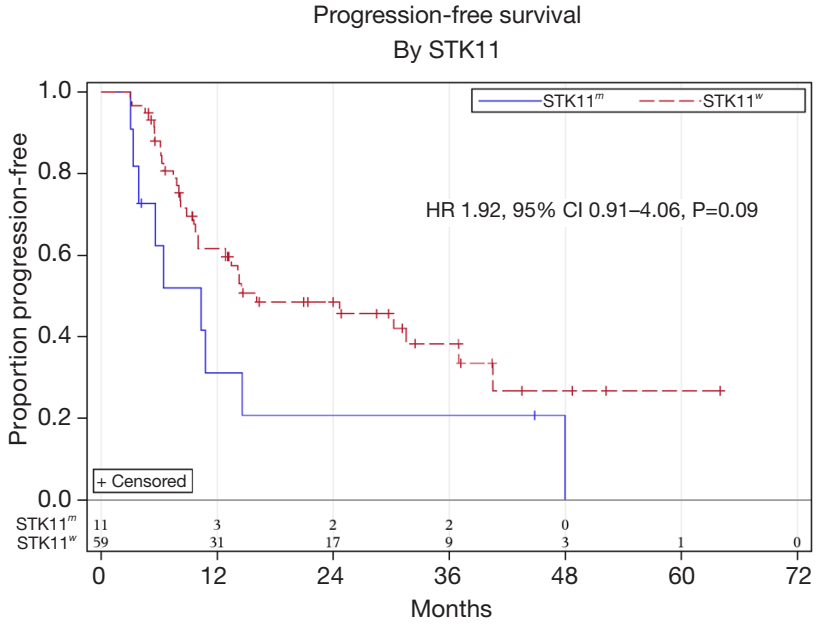

Figure 1 Progression free survival for $S T K 11^{m}$ and $S T K 11^{w}$ stage III NSCLC. NSCLC, non-small cell lung cancer.

the $S T K 11^{m}$ patients were significantly younger $(\leq 65$ years) $(\mathrm{P}=0.05)$ (Data not shown). Majority of the patients included were current/former smokers regardless of the STK11 mutational status. Regarding performance status, most of the patients included in the study had an ECOG performance status of $0-1$. Histology was divided into non-squamous and squamous NSCLC. Non-squamous histology was predominant in both the STK1 $1^{w}$ and STK1 $1^{\mathrm{m}}$ groups comprising $62.7 \%$ and $72.7 \%$, respectively, without noticing statistically significant difference $(\mathrm{P}=0.73)$. There was no significant difference in the frequency of stage IIIA versus stage IIIB/C among the groups. The frequency of KRAS or TP53 mutations were not significantly different between the STK11 groups. PD-L1 expression was $<1 \%$ in $63 \%$ of the $S T K 11^{m}$ patients, but there was no significant difference in the PD-L1 expression categories among the $S T K 11^{m}$ and $S T K 11^{w}$ groups.

\section{Treatments}

All patients included in the analysis received CCRT (Table 1). ICI consolidation was delivered to $17 / 59 S T K 11^{w}$ and $6 / 11$ $S T K 11^{m}$ patients. Of note, 20/59 STK1 $1^{w}$ and 4/11 STK1 $11^{m}$ patients were diagnosed with NSCLC after the publication of the PACIFIC trial (16) which was the first phase III randomized trial to show improvement in PFS when using consolidation durvalumab after CCRT in stage III NSCLC. The ICI consolidation given to the $6 S T K 11^{m}$ patients included pembrolizumab, which was delivered on a clinical trial, for 4 patients and durvalumab for 2 patients. All ICI delivered for the $S T K 11^{w}$ group was durvalumab.

\section{Survival outcomes}

Median follow up time was 15.7 months. Univariate analysis showed a trend towards worse PFS for STK1 $11^{m}$ compared to $S T K 11^{w}$ patients; however, this was not statistically significant (HR 1.92, 95\% CI, 0.91-4.06, $\mathrm{P}=0.09$ ) (Figure 1). There was no statistically significant difference in PFS based on sex, age, ECOG performance status, histology, stage, KRAS, TP53, PD-L1 level, or ICI consolidation (Table 2). On multivariable analysis adjusting for ECOG performance status and stage (Table 3), there was a significantly worse PFS for $S T K 11^{m}$ compared to STK1 $1^{w}$ patients (HR 2.25, $95 \%$ CI, $1.03-4.88, \mathrm{P}=0.04)$. OS by univariate analysis was not statistically different for the STK $11^{m}$ vs. STK $11^{w}$ (HR 1.47, 95\% CI, 0.49-4.38, P=0.49) (Figure 2).

\section{Discussion}

To our knowledge, this is the first study to explore $S T K 11^{m}$ as a prognostic mutation in stage III NSCLC patients who received CCRT. Our results showed that PFS, after adjusting for performance status and cancer stage, was worse for stage III NSCLC patients who had STK1 $1^{m}$ compared to $S T K 11^{w}$ (HR 2.25, 95\% CI, 1.03-4.88). Although data in the literature showed association of $S T K 11^{m}$ with poor outcomes in advanced stage NSCLC $(9,11,15)$, its impact on stage III NSCLC has not been established. In a study examining the prognostic impact of $S T K 11^{\mathrm{m}}$ in stage I-IV NSCLC (10), 21 patients had stage III disease. The median OS for all patients included was worse for the $S T K 11^{m}$ compared to $S T K 11^{w}$ patients (24 vs. 69 months, $\mathrm{P}=0.005$ ), but there were no survival outcomes reported specifically for the stage III NSCLC patients (10). Another retrospective analysis which included 23 patients with a mix of stage IIIB and IV NSCLC also did not identify the stage III cases as a subgroup for analysis (17). Our study included only stage III NSCLC patients who received CCRT with or without ICI consolidation.

The effect of $S T K 11^{m}$ on benefit from ICI consolidation post CCRT in stage III NSCLC could not be concluded in our study due to the small sample size. In advanced stage NSCLC, STK1 $1^{m}$ was associated with low PDL1 expression and resistance to PD-1/PD-L1 ICIs (11). However, the association of $S T K 11^{m}$ and poor response to ICI is still debatable as other reports showed good response 
Table 2 Progression free survival (PFS) by univariate analysis

\begin{tabular}{|c|c|c|c|c|c|c|}
\hline \multirow{3}{*}{$\begin{array}{l}\text { Variable } \\
\text { Sex }\end{array}$} & \multirow{3}{*}{$\begin{array}{c}\text { Level } \\
\mathrm{M}\end{array}$} & \multirow{3}{*}{$\begin{array}{l}\mathrm{N} \\
35\end{array}$} & \multicolumn{4}{|c|}{ Progression-free survival } \\
\hline & & & \multirow{2}{*}{$\begin{array}{c}\text { Hazard ratio } \\
1.07\end{array}$} & \multicolumn{2}{|c|}{$95 \% \mathrm{Cl}$} & \multirow{2}{*}{$\frac{P \text { value }}{0.83}$} \\
\hline & & & & 0.58 & 1.98 & \\
\hline & $\mathrm{F}$ & 35 & Ref & - & - & \\
\hline Age & $\leq 65$ years & 41 & 1.61 & 0.83 & 3.13 & 0.16 \\
\hline \multirow[t]{2}{*}{ ECOG } & $2-3$ & 10 & 1.96 & 0.89 & 4.31 & 0.10 \\
\hline & $0-1$ & 60 & Ref & - & - & \\
\hline \multirow[t]{2}{*}{ Histology } & Non-squamous & 45 & 1.31 & 0.67 & 2.58 & 0.43 \\
\hline & Squamous & 25 & Ref & - & - & \\
\hline \multirow[t]{2}{*}{ STK11 } & Yes & 11 & 1.92 & 0.91 & 4.06 & 0.09 \\
\hline & No & 59 & Ref & - & - & \\
\hline \multirow[t]{2}{*}{ KRAS } & Yes & 14 & 1.10 & 0.50 & 2.42 & 0.81 \\
\hline & No & 56 & Ref & - & - & \\
\hline \multirow[t]{2}{*}{ TP53 } & Yes & 21 & 1.02 & 0.52 & 1.99 & 0.96 \\
\hline & No & 49 & Ref & - & - & \\
\hline \multirow[t]{2}{*}{ PD-L1 } & $<1 \%$ & 21 & 1.54 & 0.54 & 4.39 & 0.40 \\
\hline & $1-50$ & 8 & 2.27 & 0.69 & 7.52 & \\
\hline
\end{tabular}

Table 3 Progression free survival (PFS) by multivariate analysis

\begin{tabular}{lcccccc}
\hline \multirow{2}{*}{ Covariate } & \multirow{2}{*}{ Level } & $\mathrm{N}$ & \multicolumn{5}{c}{ Progression-free survival } \\
\cline { 5 - 8 } & & & Hazard ratio & $95 \% \mathrm{Cl}$ & $\mathrm{P}$ value \\
\hline ECOG & $2-3$ & 10 & 2.49 & 1.10 & 5.65 & 0.03 \\
& $0-1$ & 60 & Ref & - & - & \\
Stage & IIIB/C & 32 & 1.65 & 0.88 & 3.08 & 0.12 \\
& IIIA & 38 & Ref & - & - & \\
STK11 & Yes & 11 & 2.25 & 1.03 & 4.88 & 0.04 \\
& No & 59 & Ref & - & - & \\
\hline
\end{tabular}

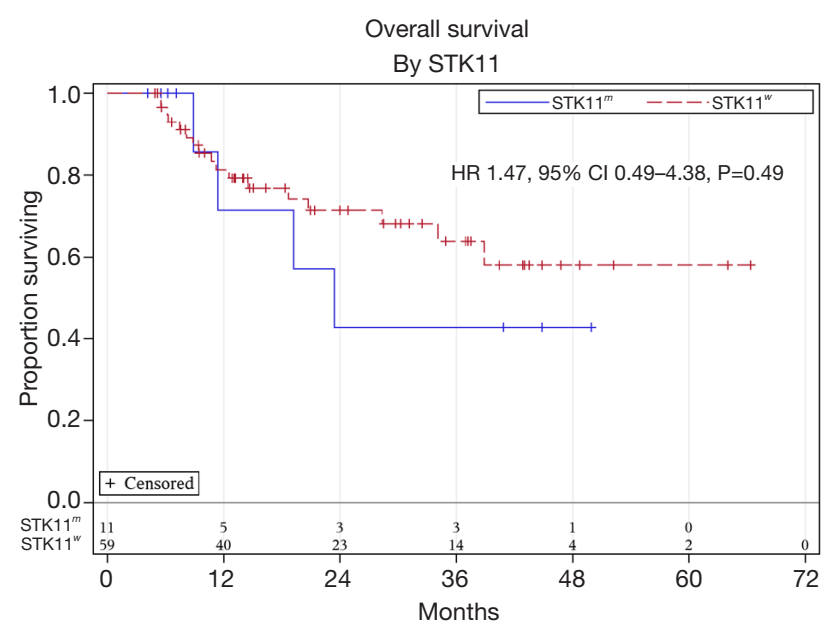

Figure 2 Overall survival for $S T K 11^{m}$ and $S T K 11^{w}$ stage III NSCLC. NSCLC, non-small cell lung cancer. 
of advanced stage $S T K 11^{m}$ NSCLC to ICI (18-20). In another driver mutation, the EGFR rather than STK11 mutation, it was questioned on retrospective studies whether there is significant benefit from durvalumab after CCRT $(21,22)$. In a cohort of 60 patients with STK1 $1^{m}$ cancers that included NSCLC and others, STK1 $1^{m}$ correlated with poor prognosis without specifically observing inferior outcomes associated with immunotherapy (23). Potential mechanisms for the $S T K 11^{m}$ resistance to ICI include impaired activity of effector $\mathrm{T}$ cells, epigenetic changes of tumor cells, modification of the cytokine and/or chemokine milieu, and diminished tumor antigenicity (11). In our study, $5 / 11$ (63\%) STK1 $11^{m}$ patients had PD-L1 $<1 \%$ and $6 / 11(55 \%)$ received ICI consolidation. The ICI consolidation used included durvalumab or pembrolizumab (pembrolizumab received on a clinical trial). No conclusions can be made about resistance of $S T K 11^{m}$ to ICI consolidation or whether a specific PD-1/PD-L1 inhibitor would function differently in the setting of $S T K 11^{m}$ in stage III NSCLC. This will need to be further studied in future trials.

The presence of other mutations that could impact the outcomes of $S T K 11^{m}$ NSCLC were also examined. Co-mutations of STK11 with either KRAS or TP53 in NSLCC have been described in advanced stage NSCLC $(12-14,24)$. Although $K R A S$ mutation was associated with better response to ICI (14), the co-mutation of STK11 and KRAS was associated with inferior survival (12-14,24). A retrospective review of patients treated with first-line therapy for metastatic NSCLC found that STK11 and $K R A S$ co-mutations were associated with worse survival compared to STK11 and TP53 co-mutation (13). In our study, only 2 patients had $S T K 11^{m}$ without co-mutation in KRAS or TP53.

Other clinical and demographic features of stage III NSCLC patients with STK1 $1^{m}$ were explored. The $S T K 11^{m}$ patients were more likely to be $\leq 65$ years old $(82 \%)$; however, there was no significant difference in age between the $S T K 11^{m}$ and $S T K 11^{w}$ patients. This is consistent with another retrospective study that examined $S T K 11^{m}$ in patients with stage I-IV non-squamous NSCLC where patients with $S T K 11^{m}$ were found to be significantly younger than $S T K 11^{w}$ patients (mean age of $58.6 \mathrm{vs} .61 .9$ years, respectively) (10). This retrospective study (10) also noted smoking to be associated with $S T K 11^{w}$ which was not seen in our study.

The authors acknowledge limitations that apply to this study. First, the retrospective nature, the small sample size, and the imbalance in the number of $S T K 11^{w}$ versus
STK1 $11^{m}$ patients are recognized. Second, the majority of patients in this study were identified from stage IV NSCLC databases who were initially diagnosed and treated for stage III disease. This might inherently reflect a selection bias of stage III NSCLC with more aggressive biology and tendency for metastases. Third, it is unknown if STK1 $1^{m}$ was present at diagnosis of stage III NSCLC or was acquired subsequently upon progression to stage IV.

\section{Conclusions}

In stage III NSCLC patients who received CCRT, STK1 $1^{m}$ was associated with worse PFS compared to $S T K 11^{w}$. Larger studies are needed to explore whether $S T K 11^{m}$ plays a role in predicting less benefit from ICI consolidation in stage III NSCLC.

\section{Acknowledgments}

Funding: Department funding by the Division of Hematology and Oncology at the University of Iowa Hospitals and Clinics.

\section{Footnote}

Reporting Checklist: The authors have completed the REMARK reporting checklist. Available at https://dx.doi. org/10.21037/tlcr-21-177

Data Sharing Statement: Available at https://dx.doi. org/10.21037/tlcr-21-177

Peer Review File: Available at https://dx.doi.org/10.21037/ tlcr-21-177

Conflicts of Interest: All authors have completed the ICMJE uniform disclosure form (available at https:// dx.doi.org/10.21037/tlcr-21-177). MF serves as an unpaid editorial board member of Translational Lung Cancer Research from Sep 2019 to Sep 2021. TK reports personal fees from AstraZeneca for consulting and advisory board, and personal fees AstraZeneca, OncLive, and Targeted Oncology for speaking. NHH's institution received grant support from BMS, Genentech, Merck on studies that in which he is the PI, and is the medical writer for UptoDate and served on a DSMB for a study sponsored by Beyond Spring. The other authors have no conflicts of interest to declare. 
Ethical Statement: The authors are accountable for all aspects of the work in ensuring that questions related to the accuracy or integrity of any part of the work are appropriately investigated and resolved. The study was conducted in accordance with the Declaration of Helsinki (as revised in 2013). The informed consent from the patients were waived. The study was approved by institutional Review Boards (IRBs) of University of Iowa Hospitals and Clinics, Indiana University Health, Northwestern Memorial Hospital, and The University of Illinois at Chicago.

Open Access Statement: This is an Open Access article distributed in accordance with the Creative Commons Attribution-NonCommercial-NoDerivs 4.0 International License (CC BY-NC-ND 4.0), which permits the noncommercial replication and distribution of the article with the strict proviso that no changes or edits are made and the original work is properly cited (including links to both the formal publication through the relevant DOI and the license). See: https://creativecommons.org/licenses/by-nc-nd/4.0/.

\section{References}

1. Antonia SJ, Villegas A, Daniel D, et al. Overall Survival with Durvalumab after Chemoradiotherapy in Stage III NSCLC. N Engl J Med 2018;379:2342-50.

2. Siegel RL, Miller KD, Jemal A. Cancer statistics, 2020. CA Cancer J Clin 2020;70:7-30.

3. Hemminki A, Markie D, Tomlinson I, et al. A serine/ threonine kinase gene defective in Peutz-Jeghers syndrome. Nature 1998;391:184-7.

4. Fang R, Zheng C, Sun $\mathrm{Y}$, et al. Integrative genomic analysis reveals a high frequency of LKB1 genetic alteration in Chinese lung adenocarcinomas. J Thorac Oncol 2014;9:254-8.

5. Shaw RJ, Kosmatka M, Bardeesy N, et al. The tumor suppressor LKB1 kinase directly activates AMP-activated kinase and regulates apoptosis in response to energy stress. Proc Natl Acad Sci U S A 2004;101:3329-35.

6. Ji H, Ramsey MR, Hayes DN, et al. LKB1 modulates lung cancer differentiation and metastasis. Nature 2007;448:807-10.

7. Koyama S, Akbay EA, Li YY, et al. STK11/LKB1 Deficiency Promotes Neutrophil Recruitment and Proinflammatory Cytokine Production to Suppress T-cell Activity in the Lung Tumor Microenvironment. Cancer Res 2016;76:999-1008.

8. Kadara H, Choi M, Zhang J, et al. Whole-exome sequencing and immune profiling of early-stage lung adenocarcinoma with fully annotated clinical follow-up. Ann Oncol 2017;28:75-82.

9. Skoulidis F, Arbour KC, Hellmann MD, et al. Association of STK11/LKB1 genomic alterations with lack of benefit from the addition of pembrolizumab to platinum doublet chemotherapy in non-squamous non-small cell lung cancer. 2019. ASCO Abstract 102

10. Pécuchet N, Laurent-Puig P, Mansuet-Lupo A, et al. Different prognostic impact of STK11 mutations in non-squamous non-small-cell lung cancer. Oncotarget 2017;8:23831-40.

11. Skoulidis F, Goldberg ME, Greenawalt DM, et al. STK11/LKB1 Mutations and PD-1 Inhibitor Resistance in KRAS-Mutant Lung Adenocarcinoma. Cancer Discov 2018;8:822-35.

12. Aredo JV, Padda SK, Kunder CA, et al. Impact of KRAS mutation subtype and concurrent pathogenic mutations on non-small cell lung cancer outcomes. Lung Cancer 2019;133:144-50.

13. Bange E, Marmarelis ME, Hwang WT, et al. Impact of KRAS and TP53 Co-Mutations on Outcomes After FirstLine Systemic Therapy Among Patients With STK11Mutated Advanced Non-Small-Cell Lung Cancer. JCO Precis Oncol 2019. doi: 10.1200/PO.18.00326.

14. Torralvo J, Friedlaender A, Achard V, et al. The Activity of Immune Checkpoint Inhibition in KRAS Mutated Nonsmall Cell Lung Cancer: A Single Centre Experience. Cancer Genomics Proteomics 2019;16:577-82.

15. Shire NJ, Klein AB, Golozar A, et al. STK11 (LKB1) mutations in metastatic NSCLC: Prognostic value in the real world. PLoS One 2020;15:e0238358.

16. Antonia SJ, Villegas A, Daniel D, et al. Durvalumab after Chemoradiotherapy in Stage III Non-Small-Cell Lung Cancer. N Engl J Med 2017;377:1919-29.

17. Facchinetti F, Bluthgen MV, Tergemina-Clain G, et al. LKB1/STK11 mutations in non-small cell lung cancer patients: Descriptive analysis and prognostic value. Lung Cancer 2017;112:62-8.

18. Qin $\mathrm{Y}, \mathrm{Yu} M$, Zhou L, et al. Durable response to combination radiotherapy and immunotherapy in EPresistant lung large-cell neuroendocrine carcinoma with B2M and STK11 mutations: a case report. Immunotherapy 2020;12:223-7.

19. Domingues I, Cedres S, Callejo A, et al. Long duration of immunotherapy in a STK11 mutated/KRAS wildtype non-small cell lung cancer patient. Pulmonology 2020;26:49-50. 
20. Nadal E, Heeke S, Benzaquen J, et al. Two Patients With Advanced-Stage Lung Adenocarcinoma With Radiologic Complete Response to Nivolumab Treatment Harboring an STK11/LKB1 Mutation. JCO Precis Oncol 2020:1239-45.

21. Aredo JV, Mambetsariev I, Hellyer JA, et al. Durvalumab for Stage III EGFR-Mutated NSCLC After Definitive Chemoradiotherapy. J Thorac Oncol 2021;16:1030-41.

22. Hellyer JA, Aredo JV, Das M, et al. Role of Consolidation Durvalumab in Patients With EGFR- and HER2-

Cite this article as: An J, Yan M, Yu N, Chennamadhavuni A, Furqan M, Mott SL, Loeffler BT, Kruser T, Sita TL, Feldman L, Nguyen R, Pasquinelli M, Hanna NH, Abu Hejleh T. Outcomes of patients with stage III non-small cell lung cancer (NSCLC) that harbor a STK11 mutation. Transl Lung Cancer Res 2021;10(8):3608-3615. doi: 10.21037/tlcr-21-177
Mutant Unresectable Stage III NSCLC. J Thorac Oncol 2021;16:868-72.

23. Krishnamurthy N, Goodman AM, Barkauskas DA, et al. STK11 alterations in the pan-cancer setting: prognostic and therapeutic implications. Eur J Cancer 2021;148:215-29.

24. La Fleur L, Falk-Sörqvist E, Smeds P, et al. Mutation patterns in a population-based non-small cell lung cancer cohort and prognostic impact of concomitant mutations in KRAS and TP53 or STK11. Lung Cancer 2019;130:50-8. 